\title{
La vaccinazione per il Covid-19 in bambini di età compresa tra i 5 e gli 11 anni
}

Il mondo pediatrico italiano si è espresso con una voce unica, con il documento che segue, sul tema della vaccinazione ai bambini di età compresa dai 5 agli 11 anni. Il Coordinamento scientifico di Medico e Bambino, che ha partecipato attivamente alla sua stesura, ne condivide pienamente i contenuti.

Si è aperto un confronto sull'opporStunità di vaccinare i bambini al di sotto dei 12 anni, e più precisamente nella fascia 5-11 anni, con il vaccino a mRNA autorizzato e raccomandato per l'uso negli Stati Uniti dalla Food and Drug Administration (FDA) (vedi l'ampia documentazione a cura dei Centers for Disease Control and Prevention $)^{1}$ qualora autorizzato per l'uso in questa fascia di età anche in Europa e in Italia.

La riflessione sul tema deve considerare quanto ci è noto circa l'epidemiologia dell'infezione da SARS-CoV-2 e le sue varianti in età pediatrica, $i$ suoi effetti clinici diretti, incluse le complicanze a breve e lungo termine, l'efficacia e la sicurezza dimostrata per il vaccino in questa fascia di età. Le valutazioni devono comprendere sia gli aspetti strettamente medici e assistenziali, che sono ovviamente prioritari, sia gli aspetti psicologici e sociali che caratterizzano la condizione dell'infanzia rispetto alla pandemia.

\section{L'INFEZIONE DA COVID NEI BAMBINI E IL PROFILO DI EFFICACIA E SICUREZZA DEL VACCINO*}

Sappiamo che l'infezione da Covid19 comporta rischi inversamente proporzionali all'età. In Italia, i dati disponibili ci dicono che fino ad oggi (9 novembre 2021, fonte Istituto Superiore di Sanità) abbiamo avuto 36 decessi in bambini e adolescenti causati da Covid-19 in 18 mesi (tasso che corrispon- de a quello registrato negli Stati Uniti, con 146 decessi tra 5 e 11 anni, pari a circa 1 su 100.000) e 206 ricoverati in Terapia Intensiva in età $<18$ anni, di cui 39 nella fascia 5-11 anni. In termini di incidenza di ricoveri in Terapia Intensiva, il valore più elevato è al di sotto dell'anno (9,9 per 100.000), dopo di che diminuisce, con valori compresi tra 0,5 e 1,5 per 100.000 nel gruppo 511 anni.

Sappiamo inoltre che l'infezione da Covid-19 è più temibile nei bambini che presentano condizioni a rischio quali: immunodeficienze (primarie o secondarie a trattamenti farmacologici, malattie oncologiche, alcune patologie croniche cardiache, renali, respiratorie), quadri severi di obesità e di diabete non adeguatamente controllato, trisomia 21 e patologie del neurosviluppo. In questi casi il rischio di complicanze (e di mortalità) da infezione da Covid-19 è risultato più elevato.

Dai dati FDA, pubblicati il 29 ottobre 2021, e ricavati dallo studio effettuato su oltre 3000 bambini 5-11 anni con il vaccino a mRNA prodotto da Pfizer, si ricava che la vaccinazione consente di prevenire il 90,7\% delle infezioni sintomatiche. Da quanto abbiamo appreso fino ad oggi, la capacità del vaccino di prevenire i ricoveri e $\mathrm{i}$ decessi è molto maggiore della sua capacità di prevenire l'infezione, quindi la vaccinazione può prevenire tutti o quasi i ricoveri in Terapia Intensiva e i decessi. Lo stesso studio ha rilevato, con un follow-up di due mesi, l'assen- za di effetti collaterali di rilievo. Vale la pena ricordare che i ricoveri in Terapia Intensiva non sono mai brevi, e che l'esperienza, terribile per gli adulti che ci sono passati, può essere ancora più devastante per un bambino.

La vaccinazione riduce di molto la complicanza indiretta dell'infezione, nota come la MIS-C o Multi-Organ Inflammatory Syndrome in Children, finora osservata con una incidenza di 3 per 10.000 soggetti sotto i 21 anni. Si tratta di un quadro infiammatorio associato all'infezione da Covid-19 che può riguardare più organi e che in molti casi comporta un interessamento cardiaco (miocardio-pericardite) che nella quasi totalità dei casi si risolve senza esiti a distanza ma che richiede un ricovero prolungato.

Sappiamo che l'incidenza di effetti collaterali nella popolazione generale, negli studi finora effettuati in fase 4 (cioè dopo la introduzione nella popolazione, e con un follow-up molto più lungo), è stata particolarmente bassa. In uno studio condotto in Israele su una popolazione molto ampia $(885.000$ soggetti vaccinati e altrettanti non vaccinati), l'unica patologia riscontrata in misura maggiore nei vaccinati (si tratta sempre di vaccino a mRNA) che nei non vaccinati è stata la miocardite, con un rapporto tra vaccinati e non di 2,7 e un'incidenza di 5 casi per $100.000^{2}$.

Questo effetto collaterale si è dimostrato comunque di modesta entità, in particolare nei giovani e negli adolescenti: lo studio che ha analizzato tutti 
i 63 casi ricoverati per miocardite comparsa in sequenza temporale con la vaccinazione anti-Covid a mRNA in 16 ospedali di riferimento americani, in soggetti di età inferiore a 21 anni, conferma che, oltre che rara, questa complicanza della vaccinazione non è severa: solo quattro casi presentavano una aritmia rilevante all'ingresso, mentre in 14 mostravano all'ecocardiografia i segni di una moderata disfunzione ventricolare; in nessun caso è stata necessaria una terapia inotropa o meccanica di supporto; nessuno è stato ricoverato in Terapia Intensiva; nessuno è deceduto e, al follow-up dopo un mese, tutte le alterazioni elettrocardiografiche ed ecocardiografiche eventualmente rilevate all'esordio erano scomparse . $^{3}$

Lo studio su efficacia e sicurezza del vaccino FDA effettuato negli USA sui bambini di età compresa tra 5 e 11 anni non ha messo in evidenza alcun caso di miocardite, ma non aveva una numerosità sufficiente a escluderne l'eventualità se molto rara. In ogni caso, in base agli studi finora effettuati su questa complicanza e in base a quanto è noto sull'epidemiologia della miocardite nella popolazione generale, si evince che il rischio di questo effetto collaterale nei bambini di 5-11 anni risulta ancora minore che negli adolescenti.

Va ricordato che il rischio di miocardite da vaccino è comunque minore del rischio di danni cardiaci da $\mathrm{Co}_{-}$ vid, inclusi quelli dovuti a MIS-C.

\section{ULTERIORI CONSIDERAZIONI SUL PROFILO BENEFICIO-RISCHIO DEL VACCINO}

Come è noto, la fascia di età $5-11$ anni è soggetta con maggiore frequenza rispetto alle età successive a infezioni delle vie aeree e alle infezioni gastrointestinali.

La comparsa di sintomatologia correlata a tali condizioni, molto spesso sovrapponibile a quella correlabile alla Covid-19, richiede attualmente di sottoporre a verifica diagnostica, tramite tampone naso-faringeo, un numero molto elevato di bambini.
L'avanzare dell'epidemia influenzale, che quest'anno si prospetta particolarmente pesante rispetto alla sua assenza dello scorso anno, amplifica il fenomeno comportando un significativo aumento del ricorso ai Servizi sanitari (pediatra di famiglia e Pronto Soccorso) con la necessità di sottoporre al tampone diagnostico in tempi rapidi di un numero elevatissimo di bambini.

Per quanto la vaccinazione riduca di molto (nel trial che ha portato all'approvazione da parte della FDA del vaccino mRNA prodotto da Pfizer, è stata del 90,7\% dopo una sola dose) ma non impedisca l'infezione da Covid-19 e la trasmissione della malattia, che resta possibile ma per un tempo significativamente minore ${ }^{4}$, in caso di malattia con sintomi compatibili può essere ridefinita, aggiornando le disposizioni in materia, la necessità di tamponi diagnostici ed esami specifici per covid nei bambini vaccinati che presentino sintomi compatibili, riducendo quindi il disagio per i bambini e le loro famiglie. Inoltre, il bambino vaccinato potrà ritenersi più sicuro e quindi più libero di partecipare ad attività sportive e associative, che ovviamente dipendono anche dalla situazione epidemiologica generale nella comunità di appartenenza.

Sulla base delle stesse evidenze, in caso di contagio in comunità (scuola ecc.) la durata dell'isolamento potrà essere ridotta nei bambini vaccinati, analogamente a quanto già previsto per gli adolescenti. Anche in questo caso potranno essere riviste le norme attuali, aumentando così i vantaggi indiretti della vaccinazione. Occorre a questo proposito ricordare che le restrizioni nei rapporti sociali e scolastici dati dalla pandemia hanno particolarmente segnato i bambini sul piano psicologico, con una vera e propria epidemia di disturbi d'ansia e disturbi del sonno, come testimoniato dagli studi condotti in Italia e in altri Paesi ${ }^{5,6}$.

Una ulteriore considerazione riguarda gli effetti a lungo termine sia dellinfezione naturale che della vaccinazione. Se è vero che ancora non sappiamo tutto degli effetti della vaccinazione a lungo termine, è anche vero che sono sempre meglio documentati gli effetti a lungo termine dell'infezione da Covid-19. Questi riguardano molti organi e sistemi, in particolare quello cardiovascolare e quello neurologico; sono inoltre ben documentati negli adulti, e forse in minor misura ma anche nei bambini e nei ragazzi nei quali sono descritti, dopo l'infezione da Covid-19, sensazione di malessere perdurante e ritiro sociale ${ }^{7.9}$. La preoccupazione rispetto all'eventualità - non documentata né per i vaccini per Covid-19 né per nessuno dei vaccini comunemente usati nell'infanzia, e nemmeno ipotizzabile su base biologica - di effetti avversi a lungo termine del vaccino va dunque comunque considerata alla luce degli effetti, questi al contrario documentati, della malattia, anche se contratta in forma lieve.

Vi è poi naturalmente un effetto positivo generale sulla circolazione del virus sia nella famiglia che nelle comunità frequentate dal bambino, anche se occorre sottolineare a questo fine quanto sia fondamentale completare la copertura vaccinale di tutta la popolazione e soprattutto di tutti i soggetti ad alto rischio.

\section{CONSIDERAZIONI CONCLUSIVE}

L'evolversi della situazione epidemiologica, e il comprensibile disorientamento delle famiglie, richiede da parte della comunità pediatrica una indicazione coerente sulla base delle informazioni a tutt'oggi disponibili.

Nel complesso, ci sentiamo di raccomandare la vaccinazione nei bambini tra 5 e 11 anni, in quanto capace di prevenire casi severi, sia pur rari, dovuti direttamente al virus o alle sue complicanze infiammatorie, di ridurre disagi per gli stessi bambini e le loro famiglie e di aumentare in generale $\mathrm{i}$ loro gradi di libertà.

La raccomandazione è ancora più forte se il bambino soffre di patologie croniche, e se convive o ha contatti stretti con adulti anziani o fragili. Le controindicazioni sono rarissime. In questi casi è opportuno rivolgersi agli specialisti che seguono il bambino. Affermiamo questo nella consapevolezza che i dati disponibili, pur essen- 
do più che sufficienti per definire l'opportunità di procedere quanto prima alla vaccinazione nei bambini di età compresa tra 5 e 11 anni, non consentono su alcuni aspetti, quali ad esempio gli effetti a lungo termine della malattia o eventuali complicanze estremamente rare della vaccinazione, di dare risposte definitive.

La raccomandazione alle famiglie non va posta in termini imperativi, ma costituisce un'opportunità di dialogo del pediatra, in particolare del pediatra di famiglia, con i genitori. La traccia che segue è un suggerimento per i pediatri nel loro dialogo con le famiglie. Contenuti e linguaggio vanno naturalmente adattati al contesto e alla relazione esistente con le famiglie.

\section{Bibliografia}

1. CDC - in: Advisory Committee on Immunization Practices' Interim Recommendation for Use of Pfizer-BioNTech COVID-19 Vaccine in Children 5-11 Years. United States, Early Release. November 5, 2021. https:// www.cdclz. gov/mmwr/volumes/70/wr/ mm7045e1.html.

2. Barda N, et al. Safety of the BNT162b2 mRNA Covid-19 Vaccine in a Nationwide Setting. N Engl J Med 2021;385:1078-90. doi: 10.1056/NEJMoa2110475.

3. Jain SS, et al. COVID-19 Vaccination-Associated Myocarditis in Adolescents. Pediatrics 2021;148(5):e2021053427. doi: 10.1542/ peds.2021-053427.

4. CDC. Delta variant: what we know about the science. https://www.cdc. gov/coronavirus/2019-ncov/variants/ delta-variant.html) 5 . Rider EA, et al. Mental health and wellbeing of children and adolescents during the Covid-19 pandemic. BMJ 2021;374:n1730. doi: 10.1136/ bmj.n1730.

6 . Bruni $\mathrm{O}$, et al. Changes in sleep patterns and disturbances in children and adolescents in Italy during the Covid-19 outbreak. Sleep Med 2021:S1389-9457(21)00094-0. doi: 10.1016/j.sleep.2021.02.003.

7. Thompson H. Children with long-covid. New Sci 2021;249(3323):10-11. doi: 10.1016/ S0262-4079(21)00303-1.

8. Buonsensi D, et al. Preliminary Evidence on Long COVID in children. Acta Paediatr 2021;110 (7):2208-2211. doi: 10.1111/ apa.15870.

9. Say D, et al. Post-acute outcomes in children with mild and asymptomatic disease. Lancet Child Adolesc Health 2021;5(6):e22e23. doi: 10.1016/S2352-4642(21)00124-3.

Il documento è stato approvato da: Società Italiana di Pediatria, Federazione Italiana Medici Pediatri, Associazione Culturale Pediatrie dalla Federazione delle Società Scientifiche e delle Associazioni di Area Pediatrica.

Sarà periodicamente aggiornato in base ai dati disponibili dalle ricerche effettuate in Italia e in ambito internazionale.

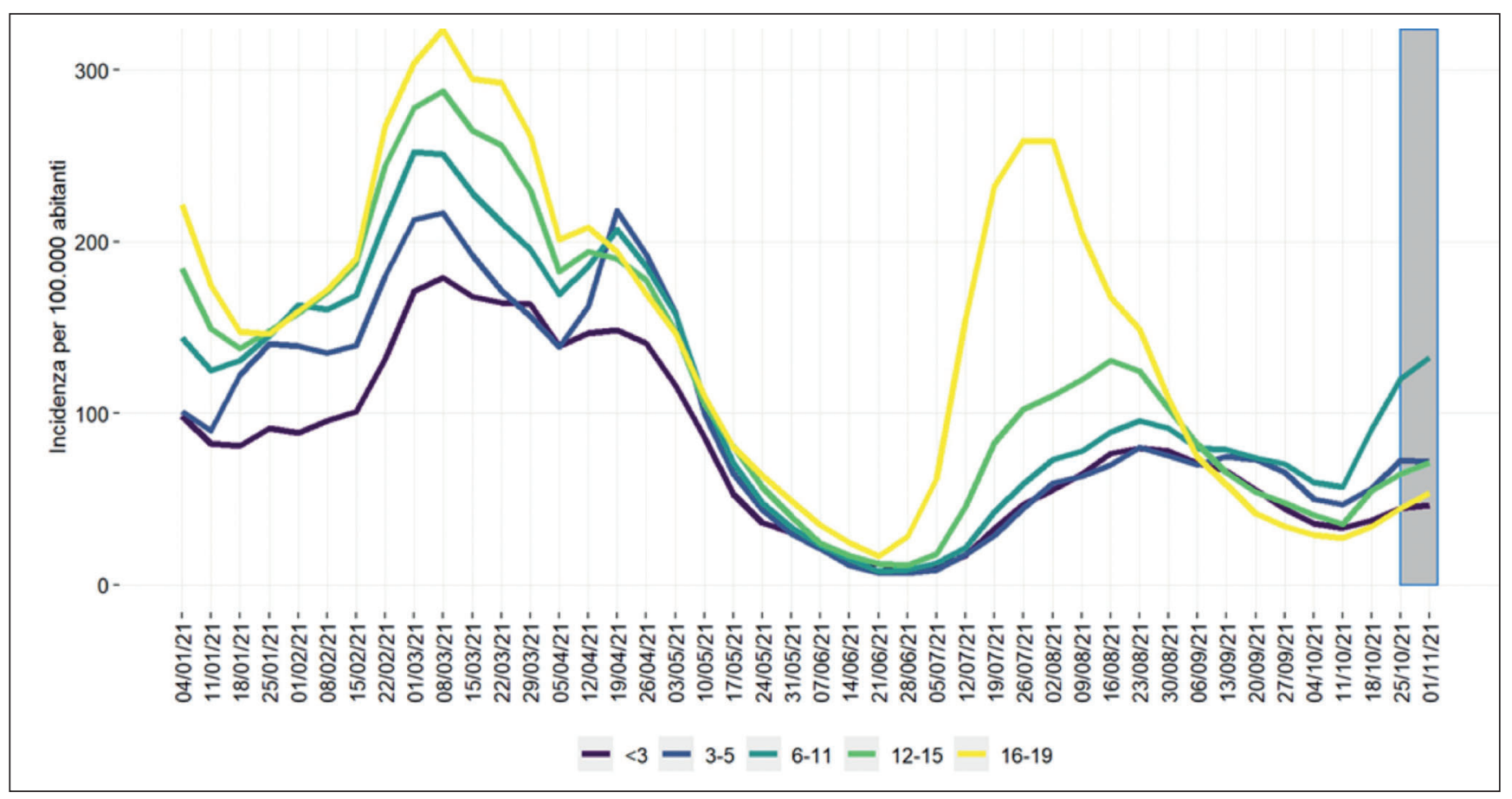

Andamento dell'infezione nei diversi gruppi di età (bambini e adolescenti). Si nota l'importante diminuzione dell'incidenza nella popolazione sottoposta a vaccinazione (linee gialle e verdi) a partire da agosto 2021 (Istituto Superiore di Sanità. Epidemia Covid-19 - Aggiornamento nazionale 10 novembre 2021). 
i

i Cari Genitori,

come certo sapete, è stato approvato in via definitiva anche per i bambini con età compresa tra i e 11 anni l'utilizzo del vaccino anti-Covid già utilizzato per gli adulti e gli adolescenti.

Tutte le Società scientifiche dei pediatri, sia in sede internazionale che in Italia, raccomandano I la vaccinazione anche dei più piccoli, sulla base degli studi condotti finora che hanno portato i le Autorità regolatorie sui farmaci e i vaccini negli Stati Uniti, in Europa e in Italia ad approvari ne l'uso in questa fascia di età.

Per contribuire a una buona informazione e a scelte consapevoli basate sulle evidenze scientifii che disponibili, vi proponiamo le seguenti considerazioni in merito alla opportunità di vaccinai zione dei bambini più piccoli.

I dati a nostra disposizione ci dicono che:

Se non si vaccina, il bambino avrà:

- Una probabilità più bassa, rispetto agli adulti, di avere dei sintomi gravi se contagiato dal virus, una probabilità dell'ordine di 3-5 su 10.000 di avere complicazioni infiammatorie serie, tali da richiedere un ricovero prolungato, e dell'ordine di 1 su 100.000 di avere una malattia così grave da richiedere cure in Terapia Intensiva. Questo se non soffre di patologie croniche (in questo caso va sempre consultato lo specialista di riferimento) per le quali il rischio è notevolmente maggiore.

- Una probabilità vicina al $100 \%$, nel corso di un anno, di dover eseguire esami diagnostici (tamponi) in caso di febbre o di altri sintomi correlabili a Covid-19 e di essere sottoposto a quarantena nel caso di positività.

\section{Se si vaccina, il bambino avrà:}

- Una probabilità dell'ordine di 3 su 100 di avere qualche sintomo, non grave (dolore locale, febbre, malessere) per uno o due giorni, e una di 1 o 2 su 100.000 di avere un effetto collaterale di maggiore entità, ma del tutto curabile.

- In caso di positività tra i suoi contatti stretti quali i compagni di scuola potrà avere tempi di quarantena ridotti (7 anziché 10 giorni).

- Sarà più libero di partecipare ad attivitò extrascolastiche e di muoversi, questo naturalmente anche in relazione alla situazione dell'infezione e alla copertura vaccinale della popolazione generale e dei suoi compagni, anche se dovrà sempre, finché saranno in vigore, rispettare le norme generali di prevenzione.

Ricordiamoci che, soprattutto per i bambini dagli 8-9 anni in su (anche se a questa età ovvia! mente sono i genitori che prendono le decisioni), i termini della questione possono essere dii scussi. Si tratta di un utile esercizio dall'indubbio valore cognitivo e civico, e un'occasione di dialogo su una questione di cui certamente hanno piacere di parlare.

A cura di: Società Italiana di Pediatria, Federazione Italiana Medici Pediatri, Associazione Culturale Pediatri e Federazione delle Società Scientifiche e delle Associazioni di Area Pediatrica. 